\title{
Hamiltonian Monte Carlo inversion
} of seismic reflection data in the acoustic approximation

\section{Other Conference Item}

Author(s):

Zunino, Andrea (D); Mosegaard, Klaus (1); Boehm, Christian; Gebraad, Lars (D); Fichtner, Andreas

Publication date:

2021

Permanent link:

https://doi.org/10.3929/ethz-b-000518163

Rights / license:

Creative Commons Attribution 4.0 International

Originally published in:

EGUsphere, https://doi.org/10.5194/egusphere-egu21-9615 
EGU21-9615, updated on 02 Dec 2021

https://doi.org/10.5194/egusphere-egu21-9615

EGU General Assembly 2021

(c) Author(s) 2021. This work is distributed under

the Creative Commons Attribution 4.0 License.

\title{
Hamiltonian Monte Carlo inversion of seismic reflection data in the acoustic approximation
}

\author{
Andrea Zunino ${ }^{1}$, Klaus Mosegaard ${ }^{2}$, Christian Boehm ${ }^{1}$, Lars Gebraad ${ }^{1}$, and Andreas Fichtner ${ }^{1}$ \\ ${ }^{1}$ ETH Zurich, Institute of Geophysics, Department of Earth Sciences, Zurich, Switzerland (andrea.zunino@erdw.ethz.ch) \\ ${ }^{2}$ University of Copenhagen, Niels Bohr Institute, Copenhagen, Denmark
}

The Hamiltonian Monte Carlo method (HMC) is gaining popularity in the geophysical community to fully address nonlinear inverse problems and related uncertainty quantification. We present here an application of HMC to invert seismic data in the acoustic approximation in the context of reflection seismology. We address a 2-D problem, in the form of a vertical cross section where both source and receivers are located near the surface of the model. To solve the forward problem we utilise the finite-difference method with PML absorbing boundary conditions. The observed data are represented by a set of shotgathers.

The crucial aspect for a successful application of the HMC lies in the capability of performing gradient computations in an efficient manner. To this end, we use the adjont state method to compute the gradient of the misfit functional, which has a computational cost of only about twice that of the forward computation, a very efficient strategy. From the collection of samples characterising the posterior distribution obtained with the HMC, we can derive quantities of interest using statistical analysis and assess uncertainties.

We illustrate an application of this methodology on a synthetic test mimicking the setup encountered in exploration problems. 